\title{
A Painful Right Testis: A Case Report
}

\author{
Saskia Weltings ${ }^{\mathrm{a}, \mathrm{c}}$, Jan H. von der Thüsen ${ }^{\mathrm{b}}$
}

\begin{abstract}
A 46-year-old Indian male without any medical history presented in the Emergency Department with a painful right testis; the pain radiated toward both flanks. Palpation of the testis was painful. There was no history of dysuria, unsafe sexual contact or weight loss. Diagnostic imaging using ultrasound revealed a suspicious mass lesion in the right testis of $2.2 \mathrm{~cm}$ diameter. The serum tumor markers alpha-fetoprotein and beta-HCG were negative, and LDH levels were normal. The patient was referred to the urology department, and a radical orchiectomy was performed. Histopathological analysis showed an adenomatoid tumor of the testicular parenchyma. No involvement of epididymis or testicular membranes was identified. Patient follow up was done every 6 months, consisting of physical examination and scrotal ultrasound. No new symptoms were present after 9 months follow up.
\end{abstract}

Keywords: Urology; Pathology; Testis tumor; Benign; Adenomatoid

\section{Introduction}

Adenomatoid tumor is a benign neoplasm, of which the histogenesis has been a point of controversy, but recent studies have provided evidence in favor of a mesothelial origin [1]. The term adenomatoid tumor was introduced by Golden and Ash in 1945 to describe a group of benign tumors with a glandular pattern and obscure histogenesis, localized in the urogenital tract.

Manuscript accepted for publication December 7, 2012

a Department of Urology, MC Haaglanden location Antoniushove, Burgemeester Banninglaan 1, 2262 BA Leidschendam, The Netherlands

${ }^{b}$ Department of Pathology, MC Haaglanden location Westeinde, Lijnbaan 32, 2512 VA The Hague, The Netherlands

${ }^{\mathrm{c} C}$ Corresponding author: Saskia Weltings, Department of Urology, MC Haaglanden location Antoniushove, Burgemeester Banninglaan 1, 2262

BA Leidschendam, The Netherlands.Email: saskiaweltings@gmail.com

doi: http://dx.doi.org/10.4021/jmc1068e
These tumors present either as an incidental finding or a slow growing scrotal mass. In men, this tumor most commonly occurs at the head of the epididymis [2]. The enlargement is usually painless with normal scrotal skin and no other symptoms. In many cases the scrotal mass has been present for several years prior to presentation. These tumors vary in size, although they most commonly measure between 1 and $2.5 \mathrm{~cm}$ in diameter (usually no larger than $5 \mathrm{~cm}$ ). The tumor prevalence is highest in males, aged between 30 and 50 years old [3].

Adenomatoid tumors are the most common tumors of paratesticular tissue, representing $30 \%$ in this group [3]. Involvement of the testicular parenchyma, as in our case, is highly exceptional. In such cases a more common primary testicular tumor (such as seminoma) may be mimicked. This makes it difficult to differentiate this benign lesion, using imaging or clinical signs, from a malignant testis tumor [4].

\section{Case Report}

A 46-year-old Indian male without any medical history presented to the Emergency Department of our hospital with a painful right testis; the pain radiated toward both flanks. Palpation of the testis was painful. There was no history of dysuria, unsafe sexual contact or weight loss. Diagnostic imaging using ultrasound, revealed a $2.2 \mathrm{~cm}$ diameter, solid, non-compressable, hyperechoic mass in the lower testicular pole, with only sparse vascularisation. There was also evidence of a possible epididymitis. The findings were not compatible with a dermoid cyst, and considered suspicious for malignancy, although atypical both in appearance and age of presentation. He was referred to the urology department. A subsequent $\mathrm{CT}$ scan of the thorax and abdomen revealed no evidence of metastases.

Serum tumor markers were checked, and alpha-fetoprotein and beta-HCG were negative. LDH was normal.

Radical orchiectomy was performed. Through an inguinal incision the testis was removed from the scrotum while the spermatic cord was being clamped, to prevent possible hematogenous metastatic spread. Postoperatively, there were no remaining complaints of pain. 


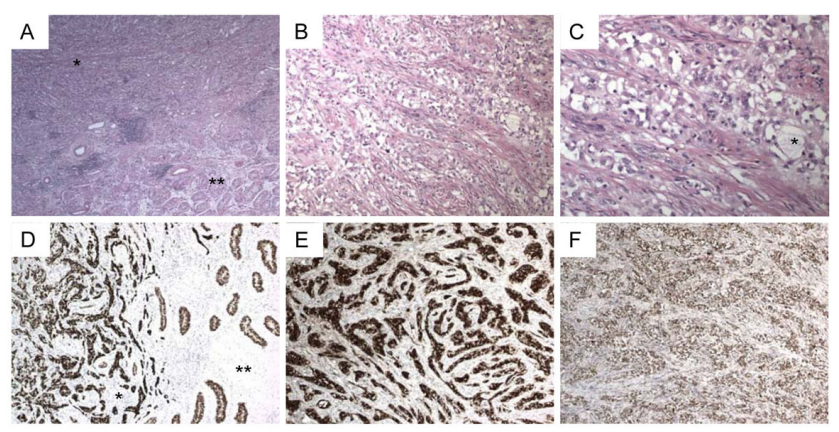

Figure 1. (Immuno) histochemical stains. A. H\&E stain $(2.5 \times)$. Tumor top left $\left(^{*}\right)$, normal testicular parenchyma bottom right $\left(^{* *}\right) ; B$. Tumor (H\&E, $10 \times)$; C. Tumor (H\&E, $20 \times)$, with glandular pattern and focal cystic change $\left(^{*}\right)$; D. Pankeratin stain $(5 \times)$. Tumor on the left $\left({ }^{*}\right)$, parenchyma on the right $\left({ }^{* *}\right)$; E. Calretinin stain $(5 \times)$; F. S100 stain $(5 \times)$.

The tumor was submitted for histopathological analysis. Macroscopic examination showed an orchiectomy specimen of $7 \times 4.5 \times 3.5 \mathrm{~cm}$, with nodular deformation. The tunica vaginalis was unremarkable. Sectioning revealed an intraparenchymal tumor with a diameter of $1.8 \mathrm{~cm}$, which was wellcircumscribed. There was no macroscopic involvement of the tunica albuginea or tunica vaginalis, or of the rete testis. The central part of the tumor showed haemorrhagic change.

Microscopically, the tumor was found to consist of an area of fibrosis with atypical epithelioid cells, arranged in strands, tubular structures and small nests. The cytoplasm was clear to amphophilic and contained moderately pleiomorphic nuclei with prominent nucleoli. Mitotic activity was limited (up to 2 mitoses per $10 \mathrm{HPF}$ ) and centrally, necrosis was seen. The abnormality was limited to the testicular parenchyma, and there was no microscopic involvement of the epididymis, or of the resection margin of the funiculus spermaticus. The surrounding parenchyma showed normal spermatogenesis. There was no evidence of an in-situ component, or of angio-invasive growth.

Immunohistochemically, the tumor cells were strongly positive for keratin AE1AE3, calretinin and S100, with focally weak staining for CD30. AFP, HCG, PLAP, inhibin and CD117 were all negative in the tumor (Fig. 1).

\section{Discussion}

Adenomatoid tumor of the testis is a benign neoplasm, which by clinical presentation is not easy to differentiate from other testicular masses. They often present as asymptomatic small lumps, which are accidentally discovered. In some cases, they are noticed with other concurrent pathology, for example a hydrocele or orchitis. Serum tumor markers are negative in all cases. In our case, the scrotal mass had probably been present for a longer period of time and was discovered during a visit to the emergency room because of pain, most likely based on a superimposed epidydimitis.

Most cases are paratesticular in location, but rare intratesticular adenomatoid tumors have been described [5]. Ultrasound is used to differentiate between intra- and extratesticular lesions and allows accurate diagnosis of cystic forms and solid lesions. However, radiographic findings are non-specific, with tumors presenting as iso-, hypo-, or hyperechoic nodules, which in most of the cases, do not allow differentiation between a benign or malignant solid intraparenchymatous lesion [6]. For intratesticular cases of adenomatoid tumor, the radiological differential diagnoses include germ cell tumors of the testis, hemangioma, malignant mesothelioma, metastatic carcinoma of the prostate, and lymphoma.

The absence of tumor markers, the well-demarcated appearance on ultrasound and age of the patient can aid in distinguishing adenomatoid tumors of the testis from other malignant scrotal tumors. In addition, excision biopsy is considered a both diagnostic and therapeutic procedure. Intraoperative frozen sections can facilitate organ sparing surgery. The aim is to prevent unnecessary orchiectomy resulting in continuation of endogenous testosterone production and preserving fertility [7].

Although follow up is advised, including physical examination and ultrasound of the scrotum, cases of metastasis or recurrence after excision have not been previously reported [2].

In conclusion, intratesticular adenomatoid tumor is a benign lesion with clinical signs similar to those of malignant testicular neoplasm. The radiographic findings are non-specific, which in most cases makes it difficult to differentiate between a benign or malignant solid intraparenchymatous lesion. If a benign lesion is suspected, it is recommended to perform intra-operative biopsy. If its benign nature is thus confirmed, the testicle can be conserved by performing tumorectomy with wide margins of resection.

\section{References}

1. Bottiger C, Koch W, Lahn C, Mehilli J, von Becker1. Schwartz EJ, Longacre TA. Adenomatoid tumors of the female and male genital tracts express WT1. Int J Gynecol Pathol. 2004;23(2):123-128.

2. Rubenstein RA, Dogra VS, Seftel AD, Resnick MI. Benign intrascrotal lesions. J Urol. 2004;171(5):17651772.

3. Amin W, Parwani AV. Adenomatoid tumor of testis. Clin Med Pathol. 2009;2:17-22.

4. Pacheco AJ, Torres JL, de la Guardia FV, Arrabal Polo MA, Gomez AZ. Intraparenchymatous adenomatoid tumor dependent on the rete testis: A case report and review of literature. Indian J Urol. 2009;25(1):126-128.

5. Alexiev BA, Xu LF, Heath JE, Twaddell WS, Phelan 
MW. Adenomatoid tumor of the testis with intratesticular growth: a case report and review of the literature. Int J Surg Pathol. 2011;19(6):838-842.

6. Feuer A, Dewire DM, Foley WD. Ultrasonographic characteristics of testicular adenomatoid tumors. J Urol. 1996;155(1):174-175.

7. Williams SB, Han M, Jones R, Andrawis R. Adenomatoid tumor of the testes. Urology. 2004;63(4):779-781. 